PHYSICAL REVIEW MATERIALS 2, 092601(R) (2018)

Rapid Communications

\title{
Scratch hardness of glass
}

\author{
Shigeki Sawamura ${ }^{1,2}$ and Lothar Wondraczek ${ }^{1, *}$ \\ ${ }^{1}$ Otto Schott Institute of Materials Research, University of Jena, Fraunhoferstrasse 6, 07743 Jena, Germany \\ ${ }^{2}$ AGC, Ltd., 1150 Hazawa-cho, Kanagawa-ku, Yokohama-shi, Kanagawa 221-8755, Japan
}

(Received 29 June 2018; published 13 September 2018)

\begin{abstract}
Since Mohs devised his prominent scale for mineral classification, the scratching behavior of inorganic solids has been intuitively related to material hardness. However, lateral deformation testing by instrumented indentation reveals a large variability in the resistance to scratch deformation relative to hardness, caused by the extent to which scratching requires higher work of deformation at given hardness due to material pileup and friction. Across a broad variety of glassy materials (with covalent, ionic, and metallic bonding), there is a strong correlation between scratch hardness and bulk modulus. Other than in crystalline materials, however, the spatial distribution of bond energy is heterogeneous on a molecular scale, so that no simple correlation exists with the mean-field average of bond energy density. Instead, inherent heterogeneity in the spatial distribution of bond energy and associated fluctuations in rigidity on a superstructural scale suggest an analogy between glasses and granular media.
\end{abstract}

DOI: 10.1103/PhysRevMaterials.2.092601

A material's response to surface mechanical contact is determined by the occurrence of lateral deformation processes, e.g., in scratch damage, abrasive wear, polishing performance, or the initiation of surface flaws in brittle solids. Our present understanding of the underlying effects is based on the intuitive assumption that there is a direct relation between the hardness and the scratch resistance of a material, both providing a measure of plastic yield [1]. This dates back to Mohs' early studies, from which the Mohs scale of hardness was derived on the basis of scratching phenomenology [2]. In contrast, later definitions of hardness were devised in quasi-isostatic conditions, describing a material's resistance to penetration with a sharp object (e.g., in classical or instrumented indentation experiments). Interestingly and especially pertinent to glasses, the discrepancy between indentation hardness and scratch hardness has been largely disregarded in the design of modern materials with improved mechanical performance: while nonisostatic surface defect generation is what limits the practical strength of glasses, indentation studies are typically employed to quantify defect resistance. For glasses, data on actual scratch hardness remains scarce and is often biased by the presence of cracking processes [3-6]. Studies which aim for quantitative measures of abrasion or polishing behavior usually follow empirical standards [7].

Here, we now apply instrumented indentation in lateral deformation mode so as to derive a quantitative measure of lateral (scratch) hardness $H_{s}$ for a wide range of glassy

\footnotetext{
*Corresponding author: lothar.wondraczek@uni-jena.de
}

Published by the American Physical Society under the terms of the Creative Commons Attribution 4.0 International license. Further distribution of this work must maintain attribution to the author(s) and the published article's title, journal citation, and DOI. materials (see Supplemental Material, Table 1 [8]). We then test $H_{s}$ against the normal hardness $H$ obtained from regular Berkovich nanoindentation, showing that there is no simple relation between both values. Lateral indentation experiments (G200, Agilent Technology Inc.) were conducted using a three-sided Berkovich diamond tip (Synton-MDP). The instrument's frame compliance and tip area function were calibrated prior to the experiments on fused silica reference glass samples (Corning 7980, Corning Inc.), using the method proposed by Oliver and Pharr [9]. We initially determined $H$ as the normal load divided by the projected contact area of the indenter tip $F_{N} / A_{c}$ on each glass specimen, performing five indentations to a depth of $2 \mu \mathrm{m}$ at a constant strain rate of $0.05 \mathrm{~s}^{-1}$. In parallel, Young's modulus $E$ was continuously recorded as a function of the indentation depth $h$ by applying a weak oscillation to the indenter tip $(\Delta h=2 \mathrm{~nm}, f=45 \mathrm{~Hz})$. Poisson ratios $v$ of all of the glasses were calculated from the longitudinal and transversal sound velocities obtained through echometry (Karl Deutsch GmbH \& Co. KG). In the lateral deformation experiments, the indenter tip was moved in edgeforward geometry across the glass surface under constant normal load while recording the scratch depth $d_{s}$ and the lateral force $F_{L}$ [5]. On each specimen, ten such constant-load scratch tests were conducted across a length of $L_{s}=200 \mu \mathrm{m}$ at a scratch speed of $50 \mu \mathrm{m} / \mathrm{s}$, for $F_{N}$ of 30-70 mN. The limited range of $F_{N}$ was chosen so as to avoid undesired tip size effects and cracking. Reference scans were conducted before and after each single test with the same Berkovich tip under a normal load of $50 \mu \mathrm{N}$ for evaluating the prescan surface level and the topography of the residual scratch groove and pileup, respectively. All experiments were conducted in laboratory air at $(296 \pm 5) \mathrm{K}$ and a relative humidity of $(60 \pm 10) \%$.

A typical dataset of $F_{L}$ as a function of lateral displacement (constant $F_{N}$ ) is depicted in Fig. 1(a). The work of deformation $W_{s}$ which is conducted to generate a scratch groove with length $L$ is derived from the integral of $F_{L} d L$ 
(a)

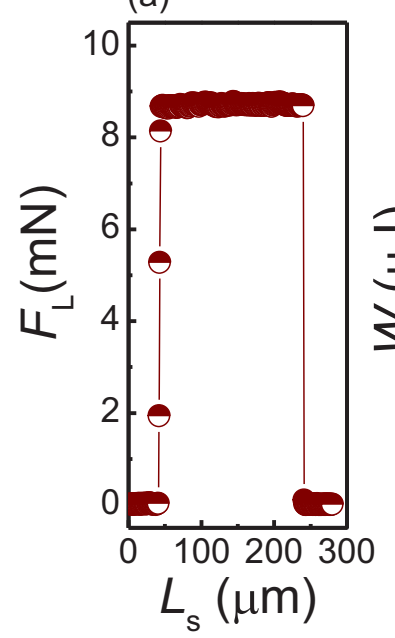

(b)

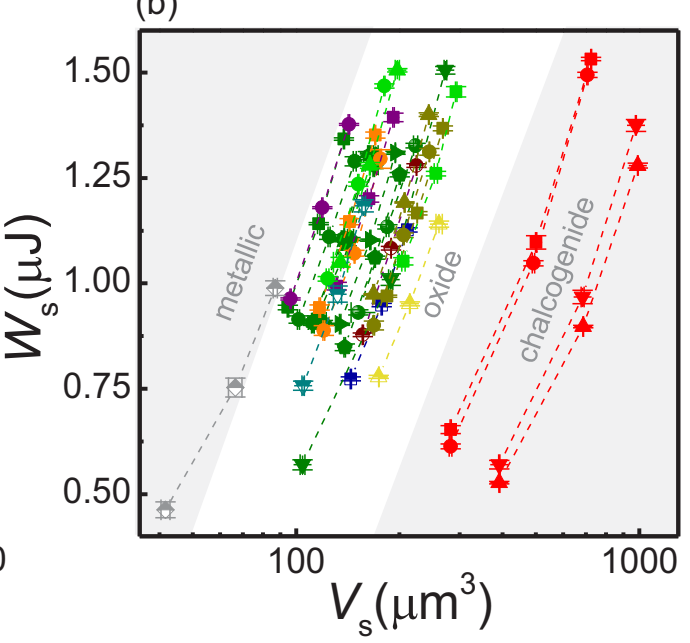

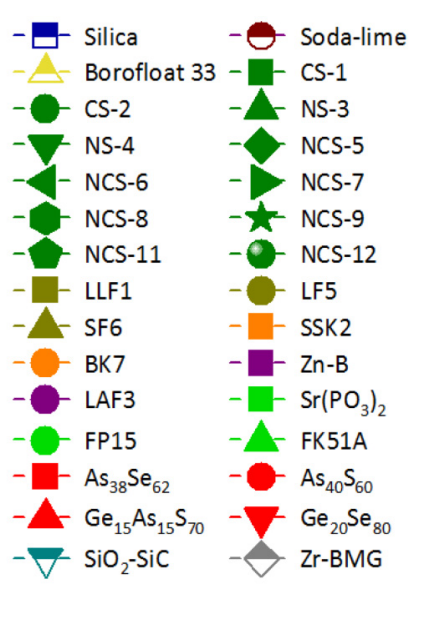

FIG. 1. Lateral load $F_{L}$ during Berkovich edge-forward scratching on a soda-lime silicate glass at a constant load of $50 \mathrm{mN}$ (a). In (b), the work of deformation $W_{s}$ is shown as a function of deformed volume (obtained through experiments with varying $F_{N}$ ) over a wide range of glassy materials.

across a scratch length of $L_{s}=100 \mu \mathrm{m}, W_{s}=\int_{100 \mu \mathrm{m}}^{200 \mu \mathrm{m}} F_{L} d L$. Corresponding data are summarized in Fig.1(b), showing the dependence of $W_{s}$ on the volume of the scratch groove $V_{s}$ (with different $V_{s}$ resulting from experiments at varying $\left.F_{\mathrm{N}}[5]\right)$. Here, the scratch volume is determined from the projected area of the indenter tip at penetration depth $d_{s}$ in scratch direction, $A_{\mathrm{tan}}, V_{s}=\int A_{\tan } d l=\int \frac{\sqrt{3} d_{s}^{2}}{2 \tan \beta} d l$, using the Berkovich edge-forward contact angle $\beta=12.95^{\circ}$. Noteworthy, this approach integrates over plastic and elastic contributions to deformation. The obtained value of $V_{s}$ differs from a value which could be obtained by ex situ topographic analysis. Within the examined range of $F_{N}$, we observed an approximately linear correlation between $W_{s}$ and $V_{s}$ over a very wide range of glass compositions [Fig. 1(b)]. This allows for extracting a load-independent (within the experimental range of $F_{N}$ ) parameter $H_{s}=d W_{s} / d V_{s}$, denoted scratch hardness. Ranging within $\sim 11.3 \mathrm{GPa}$ [zircon-based bulk metallic glass (Zr-BMG)] and 1.3 GPa [germanium-based chalcogenide glass $\left.\left(\mathrm{Ge}_{15} \mathrm{As}_{15} \mathrm{~S}_{70}\right)\right], H_{s}$ is strongly dependent on material composition (Supplemental Material, Table 1 [8]). It reflects the work which is required to deform a certain volume of material through scratching. Noteworthy, with the exception of the chalcogenide glasses in which $T / T_{g}$ approaches 0.8 [10], at room temperature, none of the considered materials reaches $T / T_{g}>0.7$ so that viscous relaxation and flow do not notably contribute to the deformation behavior [11].

Obviously and across all examined classes of glass, there is a strong, positive correlation of $H_{S}$ with bulk modulus $K$ (obtained from $v$ and $E$ ), Fig. 2(a), similar to previous observations on compacted silica and some silicate glasses $[5,6]$. We will later inspect this correlation in more detail. The atomic packing density $C_{g}$ is obtained from the ratio between the minimum theoretical volume occupied by the ions and the corresponding effective volume of the glass, $C_{g}=\rho \sum f_{i} V_{i} / \sum f_{i} M_{i}$ (with $V_{i}$ of the $i$ th constituent at molar fraction $f_{i}$ and the chemical formula $A_{x} B_{y}, V_{i}=$ $4 / 3 \pi N\left(x r_{A}^{3}+y r_{B}^{3}\right)$, the specific mass $\rho$, the Avogadro num- ber $N$, the ionic radii $r_{A}$ and $r_{B}$, and molar mass $M_{i}$ ). In addition, the mean-field bond energy density $\left\langle U_{0} / V_{0}\right\rangle$ is estimated through linear mixing from the molar dissociation enthalpies $\Delta H_{a, i}$ of all components $i[12]$,

$$
\left\langle\frac{U_{0}}{V_{0}}\right\rangle=\frac{\sum f_{i} \Delta H_{a i}}{\sum f_{i} M_{i} / \rho}
$$

The values of $\Delta H_{a, i}$ are estimated from the molar heat of formation $\Delta H_{f}$ of the component $A_{x} B_{y}$, using an ordinary Born-Haber cycle [13], $\Delta H_{a, i}=x \Delta H_{f}(A$, gas $)+$ $y \Delta H_{f}(B$, gas $)-\Delta H_{f}\left(A_{x} B_{y}\right.$, solid $)$. Noteworthy at this point, $\left\langle U_{0} / V_{0}\right\rangle$ provides access to the elastic moduli of ionic or covalent crystals with known structure. In glassy materials, it ignores the inherent absence of structural periodicity and the presence of fluctuations in bond energy density which occur on superstructural and longer scale (i.e., $1-3 \mathrm{~nm}$ ).

The correlation of $H_{s}$ with $C_{g}$ and $\left\langle U_{0} / V_{0}\right\rangle$ is much weaker as compared to that with $K$ [Figs. 2(b) and 2(c)]. Only on closer inspection, pronounced local correlations between $H_{s}$ and certain structural parameters can be identified within individual groups of glasses, for example, among the technologically relevant borosilicates, soda-lime silicates (NCS), and phosphates, respectively. Without the intention to go into detail here, such local correlations can typically be understood on the basis of the specific chemical arrangement and network topology which are pertinent to each type of glasses. For example, among the chalcogenide glasses which exhibit primarily covalent bonding, network dimensionality and cross-linking can readily be tuned between floppy, rigid, and stressed-rigid states through the presence of tetrahedral, trigonal, or chain-forming groups [14]. It has been argued that such topochemical engineering enables dedicated tailoring of material hardness under normal load [15-17]. On the other hand, such highly specific topochemical correlations do not allow for generalization across inherently different types of glassy solids such as in the present consideration. 
(a)

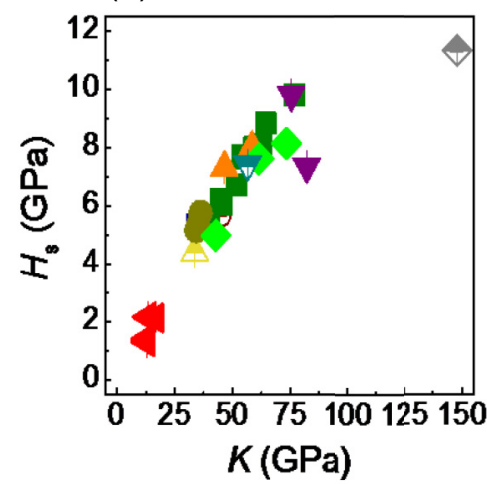

(b)

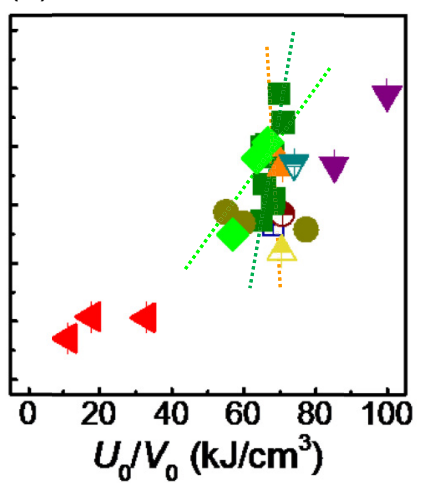

(c)

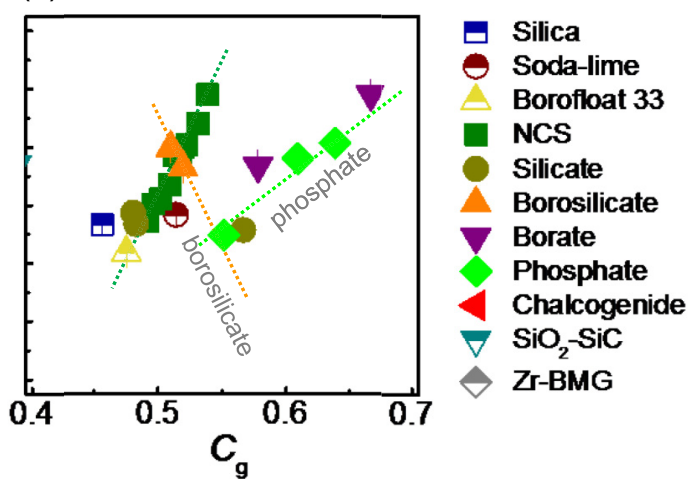

FIG. 2. Dependence of scratch hardness $H_{s}$ on bulk modulus $K$ (a), average mean-field energy density $\left\langle U_{0} / V_{0}\right\rangle$ (b), and atomic packing density $C_{g}$ (c) for a variety of glassy materials.

Generalist considerations of the normal hardness of glasses have been built on the assumption that deformation is driven by the interplay of structural compaction and shear [18]. Simplistic relations have been identified between $C_{g}$ (or compressibility, $K^{-1}$ ), Poisson's ratio, and structural dimensionality [19], from which design strategies for hardness and defect resistance can sometimes be conceived [18,20]. Translation of such assumed relations to scratch hardness appears to be significantly more complex.

As already noted, correlations between mean-field bond energy density, bulk modulus, and normal hardness are established for covalent and ionic crystals [21], but do not readily apply to glassy materials due to their inherent heterogeneity on a molecular scale. Inspecting in more detail the homogeneity of energy density, the bulk modulus $K$ is reconsidered,

$$
K=-V \frac{d p}{d V}=F\left\langle\frac{U_{0}}{V_{0}}\right\rangle .
$$

Here, the factor $F$ describes the extent to which $K$ scales with the mean-field bond energy density. For glasses, the experimentally observed $K$ is often lower than $\left\langle U_{0} / V_{0}\right\rangle$. Such $F<1$ indicates that the overall structural cohesion is lower than one may expect from the constitutive approach of Eq. (1) and the employed values of $\Delta H_{f}$. We argue that this reflects the above noted heterogeneity in bond energy distribution: the decrease in structural cohesion is a direct result of the pertinent density fluctuation and the associated presence of soft and rigid modes. This draws an analogy to granular media, in which the elastic moduli are a result of intergranular cohesion. The dependence of scratch hardness $H_{\mathrm{s}}$ on $F$ and $\left\langle U_{0} / V_{0}\right\rangle$ is shown in Fig. 3. Generally, high $H_{s}$ requires high mean-field energy density of the chemical constituents and high values of $F$. On the other hand, $H_{s}$ decreases with lower $F$ and increasing deviation from the mean-field approximate of bond energy. Taking the example of the tetrahedral network of vitreous silica, the latter may be caused by lateral fluctuations of network rigidity [22].

Per se, not only bulk modulus but also surface properties need to be considered in the evaluation of $H_{s} / H$. The lateral force used for calculating $H_{s}$ can be expressed as the sum of the plowing force $F_{p}$ and the adhesive force $F_{a}$ [23]. While $F_{p}$ describes plowing through the material, $F_{a}$ is a function of contact friction and shear. Both affect the deformation modes which underlie the creation of the scratch groove, and are affected in turn. The effective contact area depends on the type and degree of deformation, for example, through material pileup or stick-slip/slip-over reactions. The friction component is generally ignored in normal indentation experiments on glasses [24], but may reduce the amount of pileup significantly in materials with no or only little strain hardening [25].

The interaction of plowing effects, friction, and material pileup in nonisostatic loading leads to a nontrivial relation between scratch hardness and normal hardness, depicted in Fig. 4. Across different types of glass, the ratio of $H_{S} / H$ is not a constant. For example, although many borosilicates and phosphates have very similar $H$, their $H_{s}$ may vary by a factor of 2 or more. This reflects fundamental variations in the underlying deformation reactions which become much more pronounced in lateral loading as compared to the more simple (quasi-isostatic) situation of normal indentation. For

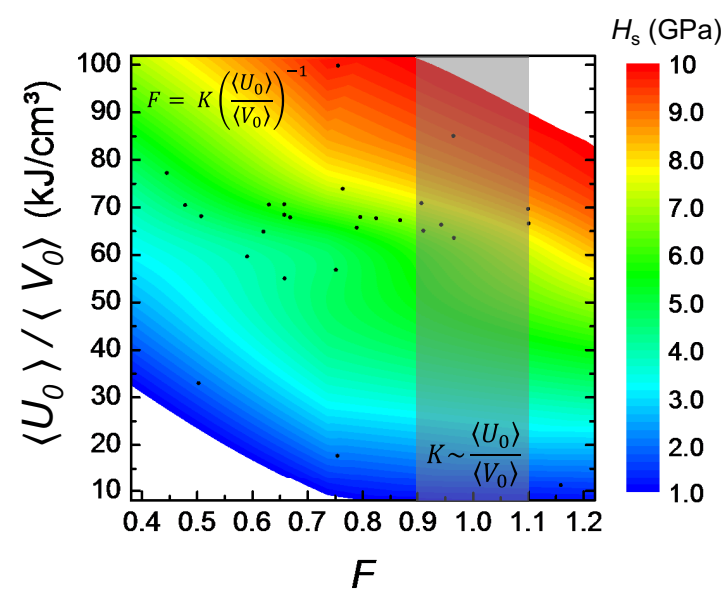

FIG. 3. Average mean-field energy density $\left\langle U_{0} / V_{0}\right\rangle$, cohesion factor $F$, and scratch hardness $H_{s}$ of glassy materials. The value of $F$ is a measure of structural heterogeneity. According to Eq. (2), it describes the deviation between bulk modulus and the mean-field estimate of bond energy density. Here, lower values of $F$ reflect increasing heterogeneity, and thus $H_{s}$ is lower than in glasses with more homogeneous spatial distribution of bond energy. 
(a)

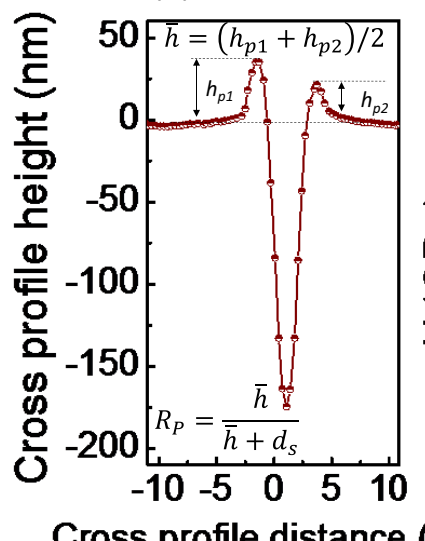

(b)

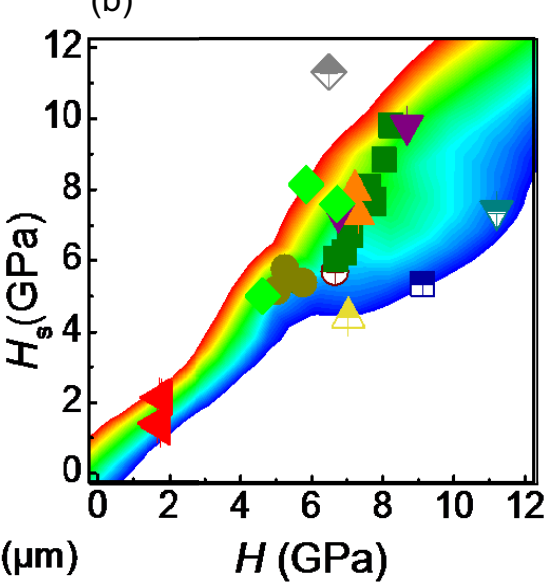

(c)

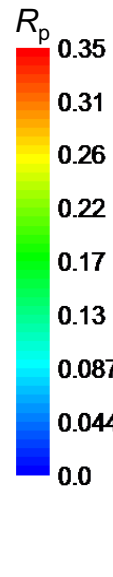

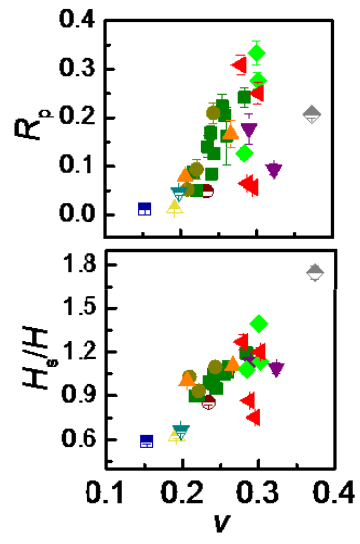

FIG. 4. Cross-profile of a typical scratch groove (soda-lime silicate glass) recorded after scratching under a normal load of $F_{N}=50 \mathrm{mN}$ (a), relation between scratch hardness $H_{s}$, normal hardness $H$, and the observed pileup ratio $R_{p}$ for $F_{N}=50 \mathrm{mN}$ (b), and dependence of $R_{p}$ and the hardness ratio $H_{s} / H$ on Poisson ratio $v(\mathrm{c})$.

example, the extent of plastic flow and the formation of pileup are strongly affected by network topology, what also reflects in a decreasing amount of pileup and decreasing $H_{s} / H$ with increasing average bond dissociation energy (Supplemental Material, Fig. S2 [8]).

A typical cross-profile scan from which the pileup ratio $R_{p}$ can be determined is provided in Fig. 4(a) (the pileup ratio is the height of material pileup in the vicinity of the scratch groove relative to the total height from the center of the scratch groove to the top of the pileup region). We find the lowest values of $R_{p}$ for glasses with low Poisson ratio [Figs. 4(b) and 4(c)], the so-called anomalous glasses such as silica or some borosilicates in which deformation is dominated by structural compaction. This is similar to normal indentation [26] and coincides with the lowest ratios of $H_{s} / H$. With increasing Poisson ratio, $R_{p}$ generally increases, but also the variability among different types of glass compositions becomes much more pronounced. This is taken as a sign of increasing structural dimensionality at intermediate Poisson ratio, where a multitude of different structural reactions may govern deformation behavior. Very similar observations have been made on related properties such as the strain-rate sensitivity of hardness and the strain-hardening exponent [10].

The variability in the ratio of $H_{s} / H$ signifies the fundamental difference between scratch hardness and normal hardness. It indicates the extent to which scratching requires higher work of deformation at given hardness. On the one hand, this is caused by differences in material pileup and friction. Both are playing a much stronger role in scratching than they do in normal indentation. On the other hand, across a broad variety of glassy materials (presenting covalent to ionic and metallic bonding), there is a strong correlation between scratch hardness and bulk modulus. Other than in crystalline materials, however, this correlation does not immediately reappear in the mean-field estimate of bond energy density. Instead, a correlation is found between $H_{s}$ and the cohesion factor $F$ : at given $\left\langle U_{0} / V_{0}\right\rangle$, greater $H_{s}$ results from greater $F$, i.e., lower deviation of the experimental bulk modulus from the mean-field estimate of bond energy density. We suggest that this is a direct result of structural heterogeneity which is inherent to glassy materials. Spatial fluctuations in bond energy density on a nanometer scale lead to fluctuations in local rigidity and, thus, structural cohesion. These manifest in lower bulk modulus, similar to granular media in which the intergranular cohesion determines the elastic properties.

This project has received funding from the European Research Council (ERC) under the European Union's Horizon 2020 research and innovation program (ERC grant UTOPES, Grant Agreement No. 681652).
[1] D. Tabor, Br. J. Appl. Phys. 7, 159 (1956).

[2] F. Mohs, Die Charaktere der Klassen, Ordnungen, and Geschlechter und Arten, oder die Charakteristik des naturhistorischen Mineral-Systemes (Arnold, Dresden, Germany, 1820), pp. $15-16$.

[3] S. Yoshida, H. Tanaka, T. Hayashi, J. Matsuoka, and N. Soga, J. Ceram. Soc. Jpn. 109, 511 (2001).

[4] E. Moayedi and L. Wondraczek, J. Non-Cryst. Solids 470, 138 (2017).
[5] S. Sawamura, R. Limbach, H. Behrens, and L. Wondraczek, J. Non-Cryst. Solids 481, 503 (2018).

[6] G. N. B. M. de Macedo, S. Sawamura, and L. Wondraczek, J. Non-Cryst. Solids 492, 94 (2018).

[7] K. H. Nielsen, S. Karlsson, R. Limbach, and L. Wondraczek, Sci. Rep. 5, 17708 (2015).

[8] See Supplemental Material at http://link.aps.org/supplemental/ 10.1103/PhysRevMaterials.2.092601 for additional data.

[9] W. C. Oliver and G. M. Pharr, J. Mater. Res. 7, 1564 (1992). 
[10] R. Limbach, B. P. Rodrigues, and L. Wondraczek, J. Non-Cryst. Solids 404, 124 (2014).

[11] M. Sakai and S. Shimizu, J. Non-Cryst. Solids 282, 236 (2001).

[12] T. Rouxel, J. Am. Ceram. Soc. 90, 3019 (2007).

[13] S. Inaba, S. Fujino, and K. Morinaga, J. Am. Ceram. Soc. 82, 3501 (1999).

[14] P. K. Gupta and J. C. Mauro, J. Chem. Phys. 130, 094503 (2009).

[15] M. M. Smedskjaer, J. C. Mauro, and Y. Yue, Phys. Rev. Lett. 105, 115503 (2010).

[16] K. Januchta, M. Bauchy, R. E. Youngman, S. J. Rzoska, M. Bockowski, and M. M. Smedskjaer, Phys. Rev. Mater. 1, 063603 (2017).

[17] M. Bauchy, Mohammad Javad Abdolhosseini Qomi, C. Bichara, F. J. Ulm, and Roland J.-M. Pellenq, Phys. Rev. Lett. 114, 125502 (2015).
[18] L. Wondraczek, J. C. Mauro, J. Eckert, U. Kühn, J. Horbach, J. Deubener, and T. Rouxel, Adv. Mater. 23, 4578 (2011).

[19] T. Rouxel, H. Ji, T. Hammouda, and A. Moréac, Phys. Rev. Lett. 100, 225501 (2008).

[20] G. N. Greaves, A. L. Greer, R. S. Lakes, and T. Rouxel, Nat. Mater. 10, 823 (2011).

[21] A. Šimůnek and J. Vackár̆, Phys. Rev. Lett. 96, 085501 (2006).

[22] O. Benzine, S. Bruns, Z. Pan, K. Durst, and L. Wondraczek, Adv. Sci. 5, 1800916 (2018).

[23] F. Wredenberg and P. Larsson, J. Mech. Mater. Struct. 2, 573 (2007).

[24] K. Hirao and M. Tomozawa, J. Am. Ceram. Soc. 70, 497 (1987).

[25] B. Taljat and G. M. Pharr, Int. J. Solid Struct. 41, 3891 (2004).

[26] S. Yoshida, S. Isono, J. Matsuoka, and N. Soga, J. Am. Ceram. Soc. 84, 2141 (2001). 